\section{Depression among older people in Bali}

Ni Wayan Suriastini ${ }^{1}$, Luh Ketut Suryani ${ }^{2}$, Bondan Sikoki ${ }^{1}$, Rodhiah Umaroh ${ }^{1}$, Hayu Qaimamunazzala ${ }^{3}$

\begin{abstract}
Introduction. Depression is the most common mental disorder. Older adults are more vulnerable to depression and are usually underdiagnosed and untreated. This study aims to determine the prevalence of depression among older adults in Bali and identify its risk factors.
\end{abstract}

Methods. This study used data from the Dementia Study in Bali 2018. Depression was assessed using a short version of the Center for Epidemiologic Studies Depression (CES-D) scale. Scores of $\geq 10$ were categorised as depression and $<10$ no depression. Risk factors included age, sex, marital status, educational level, employment status, residential district, urban status, economic status, household members, social participation, hobby activities, activities of daily living (ADL), instrumental activities of daily living (IADL), and comorbidities. Older adults with and without depression were compared to determine any risk factors. Multivariate logit regression was used to determine the association between risk factors and depression.

Results. A total of 774 female and 719 male participants aged 60 to 100 (mean, 70 \pm 7.3 ) years were included. The mean CES-D score was $9.04 \pm 5.92$ (range, $0-30$ ). Using the cut-off CES-D score of $\geq 10$ as depression, $631(42.3 \%)$ participants were considered depressed. The prevalence of depression was similar between male and female participants and increased with age. Among female older adults, depression was associated with being married (odds ratio $[\mathrm{OR}]=1.53$, $\mathrm{p}=0.019)$, ADL dependent (OR=2.58, $\mathrm{p}=0.002)$, IADL dependent $(\mathrm{OR}=1.93, \mathrm{p}=0.033)$, having high comorbidity scores $(\mathrm{OR}=3.53$, $\mathrm{p}=0.000)$, and residing in low socioeconomic districts $(\mathrm{OR}=2.53$, $\mathrm{p}=0.026)$. Whereas having a household member aged $0-5$ years $(\mathrm{OR}=0.570, \mathrm{p}=0.013)$ and being active in a hobby $(\mathrm{OR}=0.339, \mathrm{p}<0.001)$ were associated with lower likelihood of depression.

Conclusion. The prevalence of depression among older adults in Bali was high $(42.3 \%)$ and increased with age. Female older adults were more likely to be depressed when they were married, ADL and IADL dependent, and had comorbidity scores of 3-4 and $\geq 5$. Having a grandchild in the household and active participation in hobbies were associated with lower risk of depression.

Key words: Aged; Depression; Indonesia; Risk factors

\section{ORIGINAL ARTICLE}

1 SurveyMETER, Depok, Sleman, Yogyakarta, Indonesia

2 Suryani Institute, Denpasar, Bali, Indonesia

3 Department of Health Behavior, Environment, and Social Medicine, Faculty of Medicine, Public Health and Nursing, Universitas Gadjah Mada, Depok, Sleman, Yogyakarta, Indonesia
Correspondence to: Ni Wayan Suriastini, SurveyMETER, Jl. Jenengan Raya No. 109, Maguwoharjo, Depok, Sleman, Yogyakarta 55282, Indonesia. Email:

suriastini@surveymeter.org 


\section{INTRODUCTION}

According to the Riskesdas in 2018, Bali had the highest proportion of household members having a mental disorder in Indonesia. ${ }^{1}$ Bali had a high rate of suicide, and $27.4 \%$ of the cases involved older adults. ${ }^{2} 80 \%$ of the cases had mood disorder as the most prominent risk factor. ${ }^{3}$

Depression is a mental disorder characterised by feelings of sadness, fatigue, guilt, and a general loss of interest in things. It is the largest contributor to disability worldwide. ${ }^{4}$ In 2019, the World Health Organization estimated that 264 million people have depression, nearly half of them are in the South-East Asia and West Pacific regions. ${ }^{5}$ Depression may occur at any age, but older adults are at higher risk, and the prevalence peaks at age 55 to 74 years. ${ }^{4}$

The older population in Indonesia had increased from $4.5 \%$ in 1971 to $7.6 \%$ in $2010 .{ }^{6}$ The proportion is estimated to increase by $84 \%$ from 2015 to $2030 .{ }^{7}$ In Bali, the proportion of people aged $\geq 60$ years was $9.76 \%$ in $2005 .{ }^{8}$ Population growth and ageing contributed largely to a $37.6 \%$ increase in the global burden of mental and substance use disorders between 1990 and 2010. ${ }^{9}$ Improper management of mental health disorders could be costly to the system.

Factors underlying depression among older adults in developed countries have been reported..$^{10,11}$ However, there is poor understanding of depression among older people in low- and middle-income countries. This study aims to determine the prevalence of depression among older adults in Bali and to identify its risk factors and solutions.

\section{METHODS}

This study used data from the Dementia Study in Bali conducted in 2018 by SurveyMETER in collaboration with Faculty of Economic and Business Universitas Udayana, Suryani Institute, Alzheimer Indonesia, Faculty of Medicine Unika Atma Jaya and supported by Knowledge Sector Initiative. The survey interviewed older adults from eight regencies and one city in Bali. The percentage of older adults at each district was $11 \%$ to $15 \%$, except for Denpasar $(5.36 \%)$ and Badung (8.65\%). 13 villages were randomly chosen from each of the nine regencies/ city; one hamlet (Banjar) was randomly chosen from each village; and 10 households with older persons were interviewed for each cluster. ${ }^{12}$ All older persons residing in a selected household were interviewed. The total sample included 1723 older persons in 130 households. Their cognitive, mental health, morbidity, demographic, social, and economic conditions were assessed.

Depression was assessed using a short version of the Center for Epidemiologic Studies Depression (CES-D) scale. CES-D is a valid and reliable self-report questionnaire to measure depressive symptoms in the general population. ${ }^{13}$ The short version consists of 10 items, each is scored 0 to 3, depending on how often each item occurred in the past week: rarely or none ( $<1$ day), some or little of the time (1-2 days), occasionally or a moderate amount of time (3-4 days), and most or all the time (5-7 days). Scores of $\geq 10$ were categorised as depression and $<10$ no depression. ${ }^{14}$

Risk factors included age, sex, marital status, educational level, employment status, residential district, urban status (according to the Central Bureau of Statistics of Indonesia ${ }^{15}$ ), economic status, household members, social participation, hobby activities, activities of daily living (ADL), instrumental activities of daily living (IADL), and comorbidities. ${ }^{16-18}$ ADL was assessed using the 6-item Katz scale; scores of 0-4 indicate dependent, 5 slightly dependent, and 6 independent. ${ }^{19}$ IADL was assessed using the 6-item IADL scale; scores of 0-2 are categorised as dependent, 3-5 slightly dependent, and 6 independent.

Based on the self-administrated comorbidity questionnaire, ${ }^{20}$ respondents were asked if they had ever been diagnosed with any comorbidities (such as memory disorder, hypertension, diabetes, tuberculosis, asthma, other chronic pulmonary disease, cardiovascular disease, liver disease, stroke, cancer, arthritis, hypercholesterolemia, prostate disease, kidney disease, and gastrointestinal disease). They were asked whether each comorbidity limited their activities ( 1 for yes, 0 for no); the maximum score is 30 , and scores are categorised as $0,1-2,3-4$, and $\geq 5 . .^{20}$

There are sex differences in social risk factors for depression among older adults. ${ }^{21,22}$ Older adults 
with and without depression were compared using the Chi-square test to determine any risk factors. Multivariate logit regression was used to determine the association between risk factors and depression.

\section{RESULTS}

A total of 774 female and 719 male participants aged 60 to 100 (mean, 70 \pm 7.3 ) years were included. The mean CES-D score was 9.04 \pm 5.92 (range, $0-30$ ). The mean duration of schooling was 4 years. The mean per capita expenditure per month in the household was 724484 rupiahs (US\$ 51). The mean scores of ADL and IADL were $5.5 \pm 1.15$ and $4.8 \pm 1.79$, respectively. The mean comorbidity score was $2.2 \pm 2.28$ (TABLE $\mathbf{1}$ ).

Using the cut-off CES-D score of $\geq 10$ as depression, $^{14} 631$ (42.3\%) participants were considered depressed. The prevalence of depression was similar between male and female participants and increased with age. The prevalence was highest among those aged $\geq 80$ years $(56.3 \%)$, followed by the 70 to 79 years age-group (41.8\%) and the 60 to 69 years age-group (39.5\%).

In univariate analysis, depression was associated with age-group, education, having a household member aged 0 to 5 years, social participation, hobby activities, residential district, ADL, IADL, and comorbidity (TABLE 2). Comparing male and female participants with depression, the prevalence of depression was higher in male than female participants when being unmarried (49.2\% vs $40.3 \%$, $\mathrm{p}=0.045)$ and having a household member aged 0 to 5 years $(41.3 \%$ vs $31.8 \%, 0.044)$ [TABLE 2].

In multivariate logit regression, risk factors associated with depression were education level of no schooling (odds ratio $[\mathrm{OR}]=2.113, \mathrm{p}<0.001$ ) and elementary level $(\mathrm{OR}=1.816, \mathrm{p}=0.001)$, residential districts of Badung ( $\mathrm{OR}=1.745, \mathrm{p}=0.042)$, Bangli $(\mathrm{OR}=2.343, \quad \mathrm{p}=0.004)$, Karangasem $(\mathrm{OR}=2.374$, $\mathrm{p}=0.003)$, and Klungkung $(\mathrm{OR}=1.861, \mathrm{p}=0.02)$, ADL dependent $(\mathrm{OR}=2.261, \mathrm{p}<0.001)$ and slightly dependent ( $\mathrm{OR}=1.471, \mathrm{p}=0.015)$, IADL dependent $(\mathrm{OR}=1.827, \mathrm{p}=0.005)$ and slightly dependent $(\mathrm{OR}=1.528, \mathrm{p}=0.002)$, and comorbidity scores of $0-2$ $(\mathrm{OR}=1.382, \mathrm{p}=0.029), 3-4(\mathrm{OR}=1.512, \mathrm{p}=0.016)$, and $\geq 5(\mathrm{OR}=3.088, \mathrm{p}<0.001)$. Whereas being active in a hobby $(\mathrm{OR}=0.402, \mathrm{p}<0.001)$ was associated with lower likelihood of depression (TABLE 3).
TABLE 1

Sociodemographic characteristics of participants

\begin{tabular}{|c|c|}
\hline Variable & Mean $\pm S D$ (range) \\
\hline $\begin{array}{l}\text { Center for Epidemiologic Studies } \\
\text { Depression score }\end{array}$ & $9.04 \pm 5.92(0-30)$ \\
\hline Sex $($ male $=1)$ & $0.48 \pm 0.49(0-1)$ \\
\hline Age, y & $69.6 \pm 7.30(60-100)$ \\
\hline Marital status (married=1) & $0.66 \pm 0.47(0-1)$ \\
\hline Education, y & $4.42 \pm 5.72(0-21)$ \\
\hline Employment status (employed=1)) & $0.57 \pm 0.49(0-1)$ \\
\hline $\begin{array}{l}\text { Household member aged 0-5 years } \\
\text { (yes=1) }\end{array}$ & $0.21 \pm 0.40(0-1)$ \\
\hline $\begin{array}{l}\text { Household member aged } 35-55 \text { years } \\
\text { (yes=1) }\end{array}$ & $0.56 \pm 0.49(0-1)$ \\
\hline Social participation (yes $=1$ ) & $0.69 \pm 0.46(0-1)$ \\
\hline Active in hobby (yes=1) & $0.54 \pm 0.49(0-1)$ \\
\hline $\begin{array}{l}\text { Monthly household expenditure per } \\
\text { capita, IDR }\end{array}$ & $\begin{array}{l}724484 \pm 567480 \\
(29333-8430000)\end{array}$ \\
\hline Region (urban=1) & $0.50 \pm 0.50(0-1)$ \\
\hline \multicolumn{2}{|l|}{ District } \\
\hline Badung $(n=174)$ & $0.11 \pm 0.32(0-1)$ \\
\hline Bangli ( $n=165)$ & $0.11 \pm 0.31(0-1)$ \\
\hline Buleleng ( $n=154)$ & $0.10 \pm 0.30(0-1)$ \\
\hline Denpasar $(n=171)$ & $0.11 \pm 0.31(0-1)$ \\
\hline Gianyar $(n=167)$ & $0.11 \pm 0.31(0-1)$ \\
\hline Jembrana $(n=156)$ & $0.10 \pm 0.30(0-1)$ \\
\hline Karangasem $(n=174)$ & $0.11 \pm 0.32(0-1)$ \\
\hline Klungkung ( $n=169)$ & $0.11 \pm 0.32(0-1)$ \\
\hline Tabanan $(n=163)$ & $0.11 \pm 0.31(0-1)$ \\
\hline Activities of Daily Living score & $5.46 \pm 1.15(0-6)$ \\
\hline $\begin{array}{l}\text { Instrumental Activities of Daily Living } \\
\text { score }\end{array}$ & $4.77 \pm 1.79(0-6)$ \\
\hline Comorbidity score & $2.22 \pm 2.28(0-16)$ \\
\hline
\end{tabular}

Among male participants, risk factors for depression were education level of no schooling (odds ratio $[\mathrm{OR}]=2.171, \mathrm{p}=0.01$ ) and elementary level $(\mathrm{OR}=1.804, \mathrm{p}=0.012)$, residential districts of Gianyar $(\mathrm{OR}=2.237, \mathrm{p}=0.036)$, Karangasem $\quad(\mathrm{OR}=2.504$, $\mathrm{p}=0.032)$, and Klungkung $(\mathrm{OR}=2.643, \mathrm{p}=0.013)$, IADL slightly dependent (OR=1.699, $\mathrm{p}=0.009)$, and comorbidity scores of $\geq 5(\mathrm{OR}=3.310, \mathrm{p}<0.001)$. Whereas being active in a hobby (OR=0.446, $\mathrm{p}<0.001$ ) was associated with lower likelihood of depression (TABLE 3). 
TABLE 2

Prevalence of depression by sociodemographics among all, male, and female older adults in Bali

\begin{tabular}{|c|c|c|c|c|c|c|c|c|c|c|c|}
\hline \multirow[t]{3}{*}{ Variable } & \multirow[t]{3}{*}{ No. } & \multicolumn{2}{|c|}{ Depression } & \multirow[t]{3}{*}{ t-stat } & \multirow[t]{3}{*}{$p$ value } & \multicolumn{4}{|c|}{ Depression } & \multirow[t]{3}{*}{ t-stat } & \multirow[t]{3}{*}{$\mathrm{p}$ value } \\
\hline & & \multirow{2}{*}{$\begin{array}{c}\text { Yes } \\
\%(n)\end{array}$} & \multirow{2}{*}{$\frac{\text { No }}{\%(n)}$} & & & \multicolumn{2}{|r|}{ Male } & \multicolumn{2}{|c|}{ Female } & & \\
\hline & & & & & & No. & $\%(n)$ & No. & $\%(n)$ & & \\
\hline Participants & 1493 & $42.3(631)$ & 57.7 (862) & -45.14 & $<0.001$ & 719 & $42.28(304)$ & 774 & 42.25 (327) & -0.01 & 0.989 \\
\hline \multicolumn{12}{|l|}{ Age group, y } \\
\hline $60-69$ & 793 & 39.5 (313) & $60.5(480)$ & 2.33 & 0.020 & 383 & $38.9(149)$ & 410 & $40.0(164)$ & 0.32 & 0.752 \\
\hline $70-79$ & 524 & 41.8 (219) & 58.2 (305) & 0.27 & 0.787 & 261 & 43.7 (114) & 263 & 39.9 (105) & -0.87 & 0.385 \\
\hline$\geq 80$ & 176 & $56.3(99)$ & $43.7(77)$ & -4.02 & $<0.001$ & 75 & $54.7(41)$ & 101 & $57.4(58)$ & 0.36 & 0.717 \\
\hline \multicolumn{12}{|l|}{ Marital status } \\
\hline Married & 988 & $42.1(416)$ & $57.9(572)$ & 0.17 & 0.862 & 589 & $40.8(240)$ & 399 & $44.1(176)$ & 1.05 & 0.293 \\
\hline Unmarried & 505 & $42.6(215)$ & $57.4(290)$ & -0.17 & 0.862 & 130 & $49.2(64)$ & 375 & $40.3(151)$ & -1.78 & 0.045 \\
\hline \multicolumn{12}{|l|}{ Education } \\
\hline No schooling & 484 & $51.0(247)$ & $49.0(237)$ & -4.78 & $<0.001$ & 126 & $54.8(69)$ & 358 & $49.7(178)$ & -0.97 & 0.331 \\
\hline Elementary & 761 & $42.2(321)$ & $57.8(440)$ & 0.06 & 0.947 & 420 & 44.5 (187) & 341 & 39.3 (134) & -1.45 & 0.146 \\
\hline Secondary or above & 248 & $25.4(63)$ & $74.6(185)$ & 5.95 & $<0.001$ & 173 & $27.8(48)$ & 75 & $20.0(15)$ & -1.29 & 0.199 \\
\hline Employment status & & & & & & & & & & & \\
\hline Employed & 851 & $41.4(353)$ & $58.5(498)$ & 0.71 & 0.481 & 469 & $40.5(190)$ & 382 & $42.7(163)$ & 0.64 & 0.525 \\
\hline Unemployed & 642 & $43.3(278)$ & $56.7(364)$ & -0.71 & 0.481 & 250 & $45.6(114)$ & 392 & $41.8(164)$ & -0.94 & 0.349 \\
\hline Household member ac & & & & & & & & & & & \\
\hline $0-5$ & 308 & 36.7 (113) & $63.3(195)$ & 2.23 & 0.026 & 160 & $41.3(66)$ & 148 & $31.8(47)$ & -1.73 & 0.044 \\
\hline $35-55$ & 840 & $40.6(341)$ & $59.4(499)$ & 1.480 & 0.139 & 397 & $41.6(163)$ & 443 & $40.2(178)$ & -2.26 & 0.796 \\
\hline Social participation & & & & & & & & & & & \\
\hline Yes & 1035 & $40.1(415)$ & $59.9(620)$ & 2.55 & 0.011 & 527 & $40.4(213)$ & 508 & 39.7 (202) & -0.21 & 0.830 \\
\hline No & 458 & $47.2(216)$ & $52.8(242)$ & -2.55 & 0.011 & 192 & $47.4(91)$ & 266 & $47.0(125)$ & -0.09 & 0.932 \\
\hline Active in hobby & & & & & & & & & & & \\
\hline Yes & 800 & 29.9 (239) & $70.1(561)$ & 10.80 & $<0.001$ & 369 & 29.5 (109) & 431 & $30.2(130)$ & 0.19 & 0.848 \\
\hline No & 693 & $56.6(392)$ & $43.4(301)$ & -10.80 & $<0.001$ & 350 & 55.7 (195) & 343 & $57.4(197)$ & 0.45 & 0.648 \\
\hline Economic status & & & & & & & & & & & \\
\hline Poor & 598 & $43.5(260)$ & 56.5 (338) & 0.77 & 0.437 & 282 & 43.3 (122) & 316 & 43.7 (138) & 0.10 & 0.920 \\
\hline Not poor & 895 & $41.5(371)$ & 58.5 (524) & -0.77 & 0.437 & 437 & $41.6(182)$ & 458 & $41.3(189)$ & -0.12 & 0.908 \\
\hline Urban status & & & & & & & & & & & \\
\hline Rural & 746 & $45.9(343)$ & $54.0(403)$ & 2.91 & 0.004 & 355 & $44.2(157)$ & 391 & 47.6 (186) & 0.91 & 0.360 \\
\hline Urban & 747 & 38.5 (288) & 61.5 (459) & -2.91 & 0.004 & 364 & $40.4(147)$ & 383 & $36.8(141)$ & -1.00 & 0.317 \\
\hline District & & & & & & & & & & & \\
\hline Badung & 174 & $37.9(66)$ & $62.1(108)$ & 1.23 & 0.219 & 89 & $35.9(32)$ & 85 & $40.0(34)$ & 0.55 & 0.585 \\
\hline Bangli & 165 & $60.0(99)$ & $40.0(66)$ & -4.93 & $<0.001$ & 73 & 53.4 (39) & 92 & $65.2(60)$ & 1.54 & 0.123 \\
\hline Buleleng & 154 & $39.6(61)$ & $60.4(93)$ & 0.70 & 0.482 & 70 & $40.0(28)$ & 84 & 39.3 (33) & -0.09 & 0.928 \\
\hline Gianyar & 167 & 46.7 (78) & $53.3(89)$ & -1.23 & 0.218 & 82 & $51.2(42)$ & 85 & $42.4(36)$ & -1.15 & 0.254 \\
\hline Jembrana & 156 & $38.5(60)$ & $61.5(96)$ & 1.06 & 0.310 & 86 & $40.7(35)$ & 70 & 35.7 (25) & -0.63 & 0.528 \\
\hline Karangasem & 174 & $48.8(85)$ & $51.2(86)$ & -1.87 & 0.061 & 81 & $49.4(40)$ & 93 & $48.4(45)$ & -0.13 & 0.896 \\
\hline Klungkung & 169 & 49.7 (84) & $50.3(85)$ & -2.08 & 0.037 & 80 & $51.2(41)$ & 89 & $48.3(43)$ & -0.38 & 0.705 \\
\hline Tabanan & 163 & $34.9(57)$ & $65.1(106)$ & 1.98 & 0.045 & 79 & 35.5 (28) & 84 & 34.5 (29) & -0.12 & 0.903 \\
\hline Denpasar & 171 & $23.9(41)$ & 76.0 (130) & 5.18 & $<0.001$ & 79 & $24.0(19)$ & 92 & $23.9(22)$ & -0.02 & 0.983 \\
\hline Activities of daily living & & & & & & & & & & & \\
\hline Dependent & 168 & $72.0(121)$ & $28.0(47)$ & -8.48 & $<0.001$ & 72 & $69.4(50)$ & 96 & $73.9(71)$ & 0.64 & 0.521 \\
\hline Slightly dependent & 263 & $52.5(138)$ & $47.5(125)$ & -3.71 & $<0.001$ & 124 & $54.0(67)$ & 139 & $51.1(71)$ & -0.48 & 0.633 \\
\hline Independent & 1062 & $35.0(372)$ & $65.0(690)$ & 9.12 & $<0.001$ & 523 & 35.8 (187) & 539 & 34.3 (185) & -0.49 & 0.625 \\
\hline $\begin{array}{l}\text { Instrumental activities of } \\
\text { living }\end{array}$ & & & & & & & & & & & \\
\hline Dependent & 217 & $65.9(143)$ & $34.1(74)$ & -7.77 & $<0.001$ & 101 & $63.4(64)$ & 116 & $68.1(79)$ & 0.73 & 0.465 \\
\hline Slightly dependent & 445 & $49.7(221)$ & $50.3(224)$ & -3.79 & $<0.001$ & 214 & $51.9(111)$ & 231 & $47.6(110)$ & -0.89 & 0.371 \\
\hline Independent & 831 & $32.1(267)$ & $67.9(564)$ & 9.12 & $<0.001$ & 404 & $31.9(129)$ & 427 & $32.3(138)$ & 0.12 & 0.900 \\
\hline Comorbidity score & & & & & & & & & & & \\
\hline 0 & 413 & $33.4(138)$ & $66.6(275)$ & 4.30 & $<0.001$ & 212 & 32.1 (68) & 201 & $34.8(70)$ & 0.59 & 0.555 \\
\hline $1-2$ & 578 & $38.4(222)$ & $61.6(356)$ & 2.40 & 0.016 & 254 & $38.2(97)$ & 324 & $38.6(125)$ & 0.09 & 0.923 \\
\hline $3-4$ & 306 & $46.1(141)$ & 53.9 (862) & -1.51 & 0.130 & 144 & $44.4(64)$ & 162 & $47.5(77)$ & 0.53 & 0.590 \\
\hline$\geq 5$ & 196 & $66.3(130)$ & $33.7(66)$ & -7.45 & $<0.001$ & 109 & $68.8(75)$ & 87 & $63.2(55)$ & -0.82 & 0.413 \\
\hline
\end{tabular}


TABLE 3

Risk factors for depression among all, male, and female older adults in Bali

\begin{tabular}{|c|c|c|c|c|c|c|}
\hline \multirow[t]{2}{*}{ Risk factors } & \multicolumn{2}{|c|}{ All subjects $(n=1493)$} & \multicolumn{2}{|l|}{ Male $(n=719)$} & \multicolumn{2}{|c|}{ Female $(n=774)$} \\
\hline & Odds ratio $(95 \% \mathrm{Cl})$ & $\mathrm{p}$ Value & Odds ratio $(95 \% \mathrm{Cl})$ & $\mathrm{p}$ Value & Odds ratio $(95 \% \mathrm{Cl})$ & $\mathrm{p}$ Value \\
\hline \multicolumn{7}{|c|}{ Age-group, y (ref: 60-69 y) } \\
\hline $70-79$ & $0.848(0.653-1.101)$ & 0.215 & 0.979 (0.674-1.422) & 0.913 & $0.734(0.499-1.081)$ & 0.117 \\
\hline$\geq 80$ & $1.217(0.815-1.818)$ & 0.337 & $1.098(0.601-2.008)$ & 0.761 & $1.288(0.732-2.267)$ & 0.380 \\
\hline \multicolumn{7}{|c|}{ Marital status (ref: unmarried) } \\
\hline Married & $1.207(0.932-1.563)$ & 0.154 & $0.837(0.539-1.299)$ & 0.427 & $1.530(1.072-2.183)$ & 0.019 \\
\hline \multicolumn{7}{|c|}{ Education (ref: secondary or above) } \\
\hline No schooling & $2.113(1.408-3.171)$ & $<0.001$ & $2.171(1.199-3.931)$ & 0.010 & $2.617(1.280-5.349)$ & 0.008 \\
\hline Elementary & $1.816(1.257-2.624)$ & 0.001 & $1.804(1.138-2.860)$ & 0.012 & $2.090(1.048-4.169)$ & 0.036 \\
\hline \multicolumn{7}{|c|}{ Employment status (ref: unemployed) } \\
\hline Employed & $1.177(0.911-1.522)$ & 0.212 & $1.083(0.731-1.606)$ & 0.691 & $1.224(0.856-1.751)$ & 0.268 \\
\hline \multicolumn{7}{|c|}{ Household member aged 0-5 y (ref: no) } \\
\hline Yes & $0.760(0.565-1.023)$ & 0.070 & $0.951(0.627-1.440)$ & 0.811 & $0.570(0.366-0.888)$ & 0.013 \\
\hline \multicolumn{7}{|c|}{ Household member aged 35-55 y (ref: no) } \\
\hline Yes & $0.850(0.656-1.101)$ & 0.217 & $0.912(0.631-1.318)$ & 0.623 & $0.816(0.560-1.190)$ & 0.292 \\
\hline \multicolumn{7}{|c|}{ Social participation (ref: no) } \\
\hline Yes & $1.067(0.808-1.409)$ & 0.646 & $1.107(0.727-1.685)$ & 0.635 & $1.047(0.713-1.538)$ & 0.815 \\
\hline \multicolumn{7}{|l|}{ Hobby (ref: not active) } \\
\hline Active & $0.402(0.315-0.513)$ & $<0.001$ & $0.446(0.315-0.631)$ & $<0.001$ & $0.339(0.235-0.488)$ & $<0.001$ \\
\hline \multicolumn{7}{|c|}{ Economic status (ref: poor) } \\
\hline Not poor & $1.093(0.852-1.402)$ & 0.484 & $1.104(0.766-1.591)$ & 0.595 & $1.116(0.785-1.586)$ & 0.541 \\
\hline \multicolumn{7}{|l|}{ Region (ref: rural) } \\
\hline Urban & $1.068(0.823-1.385)$ & 0.620 & $1.151(0.789-1.679)$ & 0.464 & $1.026(0.707-1.489)$ & 0.892 \\
\hline \multicolumn{7}{|l|}{ District (ref: Denpasar) } \\
\hline Badung & 1.745 (1.021-2.982) & 0.042 & $1.873(0.862-4.066)$ & 0.113 & $1.603(0.742-3.462)$ & 0.229 \\
\hline Bangli & $2.343(1.314-4.178)$ & 0.004 & $2.061(0.884-4.807)$ & 0.094 & $2.537(1.116-5.767)$ & 0.026 \\
\hline Buleleng & $1.684(0.965-2.938)$ & 0.066 & 1.939 (0.843-4.457) & 0.119 & $1.515(0.700-3.283)$ & 0.292 \\
\hline Gianyar & $1.491(0.882-2.519)$ & 0.136 & $2.237(1.054-4.749)$ & 0.036 & $0.956(0.448-2.044)$ & 0.908 \\
\hline Jembrana & $1.266(0.725-2.210)$ & 0.407 & $1.757(0.789-3.917)$ & 0.168 & $0.843(0.372-1.914)$ & 0.684 \\
\hline Karangasem & $2.374(1.350-4.175)$ & 0.003 & $2.504(1.082-5.792)$ & 0.032 & $2.198(0.995-4.855)$ & 0.051 \\
\hline Klungkung & $1.861(1.102-3.144)$ & 0.020 & $2.643(1.226-5.700)$ & 0.013 & $1.303(0.619-2.742)$ & 0.485 \\
\hline Tabanan & $1.063(0.605-1.866)$ & 0.831 & $1.341(0.591-3.042)$ & 0.483 & $0.865(0.390-1.919)$ & 0.721 \\
\hline \multicolumn{7}{|c|}{$\begin{array}{l}\text { Activities of Daily Living score (ref: } \\
\text { independent) }\end{array}$} \\
\hline Dependent & $2.261(1.475-3.464)$ & $<0.001$ & $1.784(0.955-3.334)$ & 0.069 & $2.580(1.413-4.712)$ & 0.002 \\
\hline Slightly dependent & $1.471(1.079-2.006)$ & 0.015 & $1.542(0.980-2.428)$ & 0.061 & $1.429(0.920-2.220)$ & 0.112 \\
\hline \multicolumn{7}{|c|}{$\begin{array}{l}\text { Instrumental activities of daily living score } \\
\text { (ref: independent) }\end{array}$} \\
\hline Dependent & $1.827(1.203-2.774)$ & 0.005 & $1.720(0.936-3.161)$ & 0.081 & $1.927(1.056-3.516)$ & 0.033 \\
\hline Slightly dependent & $1.528(1.163-2.008)$ & 0.002 & $1.699(1.142-2.527)$ & 0.009 & $1.394(0.937-2.075)$ & 0.101 \\
\hline \multicolumn{7}{|c|}{ Comorbidity score (ref: 0) } \\
\hline $0-2$ & $1.382(1.034-1.847)$ & 0.029 & $1.435(0.945-2.179)$ & 0.090 & $1.427(0.935-2.179)$ & 0.100 \\
\hline $3-4$ & $1.512(1.081-2.114)$ & 0.016 & $1.336(0.828-2.157)$ & 0.236 & $1.936(1.185-3.163)$ & 0.008 \\
\hline$\geq 5$ & 3.088 (2.070-4.607) & $<0.001$ & $3.310(1.905-5.751)$ & $<0.001$ & $3.531(1.919-6.498)$ & $<0.001$ \\
\hline
\end{tabular}


Among female participants, risk factors for depression were being married $(\mathrm{OR}=1.530$, $\mathrm{p}=0.019$ ), education level of no schooling (odds ratio $[\mathrm{OR}]=2.617, \mathrm{p}=0.008$ ) and elementary level $(\mathrm{OR}=2.090, \mathrm{p}=0.036)$, residential district of Bangli $(\mathrm{OR}=2.537, \mathrm{p}=0.026)$, ADL dependent $(\mathrm{OR}=2.580$, $\mathrm{p}=0.002)$, IADL dependent (OR=1.927, $\mathrm{p}=0.033)$, and comorbidity scores of $3-4(\mathrm{OR}=1.936, \mathrm{p}=0.008)$ and $\geq 5(\mathrm{OR}=3.531, \mathrm{p}<0.001)$. Whereas having a household member aged $0-5$ years ( $\mathrm{OR}=0.570$, $\mathrm{p}=0.013)$ and being active in a hobby $(\mathrm{OR}=0.339$, $\mathrm{p}<0.001)$ were associated with lower likelihood of depression (TABLE 3).

\section{DISCUSSION}

Consistent with previous studies, ${ }^{21,22}$ there were slight differences in risk factors between male and female older adults in Bali. Although employment and economic status were not associated with depression, such associations have been reported in other studies. ${ }^{23-25}$ The prevalence of depression among older adults in Bali was 42.3\%, which is higher than the $31.7 \%$ in West Java, ${ }^{26} 11.3 \%$ in Yogyakarta, ${ }^{12}$ and $24.9 \%$ in the entire nation, ${ }^{27}$ as well as $14.5 \%$ in South Korea, ${ }^{23} 39.6 \%$ in Vietnam, ${ }^{28}$ and $32.9 \%$ in Thailand. ${ }^{29}$

Opposite to previous studies, ${ }^{16,18,28}$ female older adults in Bali were more likely to be depressed if they were married (compared with unmarried), probably because married women may feel overwhelmed by family responsibility such as the obligation to maintain husband's necessities in the house. ${ }^{30,31}$ In addition, female older adults in Bali were less likely to be depressed if having a grandchild in the household, consistent with a study in Greece. ${ }^{21}$ Although grand-parenting can be a burden, the bond between female older adults and grandchildren is beneficial for well-being and can reduce the feeling of loneliness in late-life. ${ }^{32}$

The impact of no or low education on depression was more noticeable in female than male older adults in Bali. Low educational status is associated with higher prevalence of depression in Korean older adults. ${ }^{33}$ Men with better education have a lower prevalence of mental disorder. ${ }^{34}$ In the United States, the prevalence of depression is higher among individuals with $<12$ years of education..$^{35}$ Each additional year of education can decrease the log odds ratio of being depressed by 3\%. ${ }^{36}$ Education plays a role in obtaining better working conditions, higher incomes, and improving the overall knowledge of health. Only $17 \%$ of our participants had higher than primary schooling.

Old adults in Bali who were active in a hobby were less likely to be depressed. High engagement in hobbies is associated with decreased mortality and depression. ${ }^{17,37}$ Staying busy with enjoyable activities provides relief to the mind. Older adults have less time and energy to worry if they keep busy with their interests. ${ }^{38}$ Singing (through a choir, karaoke, or at home) can release endorphins. ${ }^{39}$ Dancing can increase strength and balance in older adults. ${ }^{40}$ Listening to and playing music are beneficial for relaxing the body and mind. ${ }^{41}$

Neighbourhoods can be a stressor to elicit depression. ${ }^{42}$ The risk of depression was higher in male older adults living in Gianyar, Karangasem, and Klungkung and in female older adults living in Bangli, compared to Denpasar. According to Statistics Bali in 2018, ${ }^{43}$ residents of Karangasem, Klungkung, and Bangli have the lowest years of schooling, with a mean of 5.52 years, 6.8 years, and 7.4 years, respectively. They also have the lowest life expectancy of around 69.83 years and have the lowest poverty line in Bali. In rural China, poor socioeconomic status results in poor access to healthcare, particularly mental health services, resulting in difficulties in diagnosis and treatment of depression. ${ }^{24}$

Female older adults who were ADL and IADL dependent and male older adults who were IADL slightly dependent were more likely to have depression. It implied that female older adults were at higher risk of depression, consistent with a study in rural China. ${ }^{44}$ Older women engage in lower physical activity than older men, resulting in a higher reduction in ADL and a lower degree of independence. ${ }^{45}$ Dependence may generate a feeling of guilt, sadness, and self-blamed that causes depression in the long term. ${ }^{46,47}$

The risk of depression increased with higher comorbidity scores. The impact of comorbidity on depression was higher in female older adults, consistent with one study. ${ }^{48}$ Chronic diseases reduce quality of life owing to associated pain and functional impairment. ${ }^{49}$ The prevalence of 
depression is higher in patients with pain. ${ }^{50}$ The three chronic illnesses with the strongest associations to depression are all related to pain. ${ }^{51}$ Disability is a stressful condition that imposes an increased level of dependency and requires adjustments to a new way of living. ${ }^{52}$ Disability in self-care, social activities, and participation in society are associated with depression. ${ }^{53}$ People with disabilities have higher levels of secondary conditions such as chronic pain, fatigue, difficulties maintaining friends, and feeling isolated. $^{54}$

This study has limitations. Its cross-sectional design can only determine the association between risk factors and depression. It is not possible to determine causation. Longitudinal studies of the population are warranted. People living in institutions such as hospitals or nursing homes were not included; the prevalence of depression among those living in institutions is higher than those living in the community. ${ }^{55}$ The self-report nature of the study may result in reporting bias; respondents may not be truthful about symptoms related to depression.

\section{CONCLUSION}

The prevalence of depression among older adults in Bali was high (42.3\%) and increased with age. Female older adults were more likely to be depressed when they were married, ADL and IADL dependent, and had comorbidity scores of 3-4 and $\geq 5$. Having a grandchild in the household and active participation in hobbies were associated with lower risk of depression.

\section{DECLARATION}

The authors have no conflict of interest to disclose.

\section{REFERENCES}

1. Agency of Health Research and Development, Indonesia Indonesia Basic Health Research 2018 [in Indonesian]. Jakarta; 2018.

2. Octaria YC. The role of health system in suicide prevention in Bali: barriers and possibilities. Royal Tropicak Institute; 2008.

3. Kurihara T, Kato M, Reverger R, Tirta IG. Risk factors for suicide in Bali: a psychological autopsy study. BMC Public Health 2009;9:327. Crossref

4. World Health Organization. Depression and Other Common Mental Disorders Global Health Estimates. Geneva; 2017.

5. World Health Organization. Depression. https://www.who.int/ news-room/fact-sheets/detail/depression Accessed 13 April 2020.
6. UNFPA Indonesia. Indonesia on the Threshold of Population Ageing. 2014.

7. United Nations Department of Economics and Social Affairs Population Division. World Population Ageing. New York; 2015.

8. Abikusno N. The Elderly of Indonesia: Current Policy and Programmes. 2005.

9. Whiteford HA, Degenhardt L, Rehm J, Baxter AJ, Ferrari AJ, Erskine HE, et al. Global burden of disease attributable to mental and substance use disorders: findings from the Global Burden of Disease Study 2010. Lancet 2013;382:1575-86. Crossref

10. Freeman A, Tyrovolas S, Koyanagi A, Chatterji S, Leonardi M, Ayuso-mateos JL, et al. The role of socio-economic status in depression: results from the COURAGE (aging survey in Europe). BMC Public Health 2016;16:1098. Crossref

11. Inaba A, Thoits PA, Ueno K, Gove WR, Evenson RJ, Sloan M. Depression in the United States and Japan: gender, marital status, and SES patterns. Soc Sci Med 2005;61:2280-92. Crossref

12. Suriastini W, Turana $Y$, Suryani LK, et al. Report of the Dementia Study in Bali 2018: Encouraging the Emergence of a Policy for Sustainability [in Indonesian]. http://www.surveymeter.org/id/ node/374 Accessed 13 April 2020.

13. Radloff LS. The CES-D Scale: a self-report depression scale for research in the general population. Appl Psychol Meas 1977;1:385-401. Crossref

14. Andresen EM, Malmgren JA, Carter WB, Patrick DL. Screening for depression in well older adults: evaluation of a short form of the CES-D (Center for Epidemiologic Studies Depression Scale). Am J Prev Med 1994;10:77-84. Crossref

15. Central Bureau of Statistics of Indonesia. Head of the Central Statistics Agency Regulation No. 37 of 2010 on Classification of Urban and Rural Areas in Indonesia [in Indonesian].

16. Liu Q, Cai H, Yang LH, Xiang YB, Yang G, Li H, et al. Depressive symptoms and their association with social determinants and chronic diseases in middle-aged and elderly Chinese people. Sci Rep 2018;8:3841. Crossref

17. Lee SH, Kim YB. Which type of social activities may reduce cognitive decline in the elderly? A longitudinal population-based study. BMC Geriatr 2016;16:165. Crossref

18. Singh A, Kaushal SK, Misra SK, Agrawal R, SinhaV. Magnitude of depression and its determinants among older persons in urban community of Agra. Indian J Community Health 2018;30:307.

19. Katz S, Ford AB, Moskowitz RW, Jackson BA, Jaffe MW. Studies of illness in the aged. The index of ADL: a standardized measure of biological and psychosocial function. JAMA 1963;185:9149. Crossref

20. Sangha O, Stucki G, Liang MH, Fossel AH, Katz JN. The SelfAdministered Comorbidity Questionnaire: a new method to assess comorbidity for clinical and health services research. Arthritis Rheum 2003;49:156-63. Crossref

21. Carayanni V, Stylianopoulou C, Koulierakis G, Babatsikou F, Koutis $\mathrm{C}$. Sex differences in depression among older adults: are older women more vulnerable than men in social risk factors? The case of open care centers for older people in Greece. Eur J Ageing 2012;9:177-86. Crossref

22. Girgus JS, Yang K, Ferri CV. The gender difference in depression: are elderly women at greater risk for depression than elderly men? Geriatrics (Basel) 2017;2:35. Crossref

23. Kim EK, Kim HO, ParkYM, Park CJ,Yu DS, Lee JY. Prevalence and risk factors of depression in geriatric patients with dermatological diseases. Ann Dermatol 2013;25:278-84. Crossref

24. Fang M, Mirutse G, Guo L, Ma X. Role of socioeconomic status and housing conditions in geriatric depression in rural China: a cross-sectional study. BMJ Open 2019;9:e024046. Crossref

25. Paul NSS, Ramamurthy PH, Paul B, Saravanan M, Santhosh SR, Fernandes D, et al. Depression among geriatric population; the need for community awareness. Clin Epidemiol Glob Health 
2019;7:107-10. Crossref

26. Hanum L, Utojoyo DB, Jaya ES. Indonesian older adults' mental health: an overview. Psychol Res Urban Soc 2018;1:74-80. Crossref

27. Madyaningrum E, Chuang YC, Chuang KY. Factors associated with the use of outpatient services among the elderly in Indonesia. BMC Health Serv Res 2018;18:707. Crossref

28. Giang LT, Nguyen TT, Tran NTT. Factors associated with depression among older people in Vietnam. Warasan Prachakon Lae Sangkhom 2019;27:181-94. Crossref

29. Chaiut W, Ruanjai T, Trongsakul S, Tamornpark R, Apidechkul T. Prevalence and factors associated with depression among the hill tribe elderly population, Thailand. J Med Assoc Thai 2018;101:977-85.

30. Carlson K, Eisenstat S, Ziporyn T. The New Harvard Guide to Women's Health. Bibliovault OAI Repository, University of Chicago Press; 2004. Crossref

31. Carter P, Uppal H, Chandran S, Potluri R. Married patients with modifiable cardiovascular risk factors have lower mortality rates. Heart 2017;103(Suppl 5):A68-9. Crossref

32. Hughes ME, Waite LJ, LaPierre TA, Luo Y. All in the family: the impact of caring for grandchildren on grandparents' health. J Gerontol B Psychol Sci Soc Sci 2007;62:S108-19. Crossref

33. Oh DH, Kim SA, Lee HY, Seo JY, Choi BY, Nam JH. Prevalence and correlates of depressive symptoms in Korean adults: results of a 2009 Korean community health survey. J Korean Med Sci 2013;28:128-35. Crossref

34. Ploubidis GB, Grundy E. Later-life mental health in Europe: a country-level comparison. J Gerontol B Psychol Sci Soc Sci 2009;64:666-76. Crossref

35. Kessler RC, Berglund P, Demler O, Jin R, Koretz D, Merikangas $\mathrm{KR}$, et al. The epidemiology of major depressive disorder: results from the National Comorbidity Survey Replication (NCS-R). JAMA 2003;289:3095-105. Crossref

36. Lorant V, Deliège D, Eaton W, Robert A, Philippot P, Ansseau M. Socioeconomic inequalities in depression: a meta-analysis. Am J Epidemiol 2003;157:98-112. Crossref

37. Tomioka K, Kurumatani N, Hosoi H. Relationship of having hobbies and a purpose in life with mortality, activities of daily living, and instrumental activities of daily living among community-dwelling elderly adults. J Epidemiol 2016;26:36170. Crossref

38. Edwards DF. Active aging: benefits of an active lifestyle on health and well-being in later life. Department of KinesiologyOccupational Therapy; 2011.

39. Dunbar RI, Kaskatis K, MacDonald I, Barra V. Performance of music elevates pain threshold and positive affect: implications for the evolutionary function of music. Evol Psycol 2012;10:688702. Crossref

40. Granacher U, Muehlbauer T, Bridenbaugh SA, Wolf M, Roth R, GschwindY, et al. Effects of a salsa dance training on balance and strength performance in older adults. Gerontology 2012;58:30512. Crossref

41. Trappe HJ. The effects of music on the cardiovascular system and cardiovascular health. Heart 2010;96:1868-71. Crossref

42. Blair A, Ross NA, Gariepy G, Schmitz N. How do neighborhoods affect depression outcomes? A realist review and a call for the examination of causal pathways. Soc Psychiatry Psychiatr Epidemiol 2014;49:873-87. Crossref

43. Badan Pusat Statistik Provinsi Bali. Statistik Daerah Provinsi Bali 2018. Bali; 2018.

44. Fang M, Chen J, Guo L, Ma X. Gender differences in geriatric depressive symptoms in rural China: the role of physical housing environments and living arrangements. Int J Environ Res Public Health 2019;16:774. Crossref

45. Overdorf V, Kollia B, Makarec K, Alleva Szeles C. The relationship between physical activity and depressive symptoms in healthy older women. Gerontol Geriatr Med 2016;2:2333721415626859. Crossref

46. Zhang AZ, Wang QC, Huang KM, Huang JG, Zhou CH, Sun $\mathrm{FQ}$, et al. Prevalence of depression and anxiety in patients with chronic digestive system diseases: a multicenter epidemiological study. World J Gastroenterol 2016;22:9437-44. Crossref

47. Kiosses DN, Alexopoulos GS. IADL functions, cognitive deficits, and severity of depression: a preliminary study. Am J Geriatr Psychiatry 2005;13:244-9. Crossref

48. Padayachey U, Ramlall S, Chipps J. Depression in older adults: prevalence and risk factors in a primary health care sample. South African Fam Pract 2017;59:61-6. Crossref

49. Katon WJ. Clinical and health services relationships between major depression, depressive symptoms, and general medical illness. Biol Psychiatry 2003;54:216-26. Crossref

50. Bair MJ, Robinson RL, Katon W, Kroenke K. Depression and pain comorbidity: a literature review. Arch Intern Med 2003;163:243345. Crossref

51. Patten SB. Long-term medical conditions and major depression in a Canadian population study at waves 1 and 2. J Affect Disord 2001;63:35-41. Crossref

52. Noh JW, Kwon YD, Park J, Oh IH, Kim J. Relationship between physical disability and depression by gender: a panel regression model. PLoS One 2016;11:e0166238. Crossref

53. Verhaak PFM, Dekker JH, de Waal MWM, van Marwijk HWJ, Comijs HC. Depression, disability and somatic diseases among elderly. J Affect Disord 2014;167:187-91. Crossref

54. Kinne S, Patrick DL, Doyle DL. Prevalence of secondary conditions among people with disabilities. Am J Public Health 2004;94:443-5. Crossref

55. Jongenelis $\mathrm{K}$, Pot AM, Eisses AM, Beekman AT, Kluiter $H$, Ribbe MW. Prevalence and risk indicators of depression in elderly nursing home patients: the AGED study. J Affect Disord 2004;83:135-42. Crossref 